\title{
Oberflächenveredler zwischen Pandemie und Transformation
}

\author{
Um sich ein Bild über die aktuelle Situation der Branche zu machen, führt der VOA regelmäßig \\ Umfragen unter seinen Mitgliedsunternehmen durch, zuletzt im Dezember 2020. \\ Das Fazit: Trotz Einschränkungen geht es der Branche derzeit den Umständen entsprechend gut.
}

Themen wie der European Green Deal, Nachhaltigkeit und der voranschreitende Strukturwandel beschäftigten die Industrie zu Beginn des letzten Jahres. Dann kam die Corona-Pandemie: Nach dem ersten Lockdown im Frühahr 2020 wurden auf allen Märkten weltweit kräftige Einbrüche verzeichnet. Um sich ein Bild über die jeweils aktuelle Situation der OberflächenBranche zu machen, führt der Verband für die Oberflächenveredelung von Aluminium e. V. (VOA) regelmäßig Umfragen unter seinen Mitgliedsunternehmen durch. Bei der letzten Umfrage im Dezember 2020 stuften $90 \%$ der befragten Unternehmen die Auswirkungen der Corona-Krise auf die Produktion als gering bis mittelmäßig ein. Ebenfalls erfreulich ist, dass $95 \%$ die Entwicklung ihres Unternehmens für das Jahr 2021 positiv bis mittelmäßig einschätzen. Zwar gaben die Teilnehmer der Umfrage einen Rückgang bei der Kapazitätsauslastung (14\%), beim erwarteten Umsatz (15\%) und bei den Auftragseingängen (18\%) an,

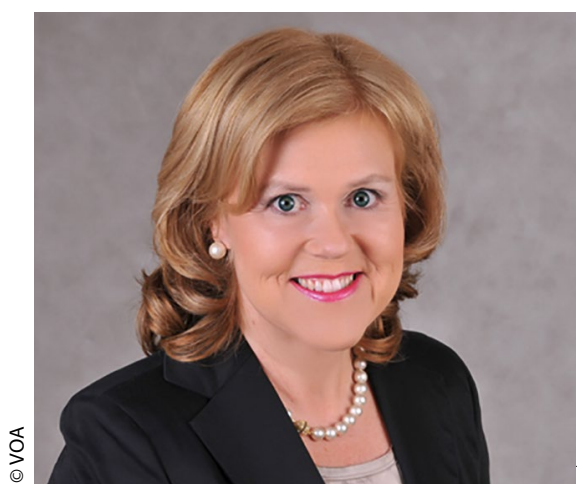

Dr. Alexa Becker, Geschäftsführerin des VOA: „Wirtschaftlichkeit und soziale Verträglichkeit sollten vor dem Hintergrund der Corona-Krise mehr denn je Hand in Hand gehen." scheinbar fielen die Einbußen im Dezember aber geringer aus, als noch im Mai erwartet wurde.

Dass die Mitarbeiter in der Branche bislang nicht von einer Kündigungswelle überrollt wurden, ist ebenfalls positiv zu bewerten. So gab es bei $85 \%$ der Unternehmen keine betriebsbedingten Kündigungen im Jahr 2020. Die Kurzarbeit hat sich bisher als wertvolles Instrument für die Unternehmens- und Beschäftigungssicherung von qualifiziertem Personal erwiesen. 30 \% der befragten Mitgliedsunternehmen nutzen diese Möglichkeit für durchschnittlich ein Drittel ihrer Mitarbeiter. Die Arbeitszeit wurde im Mittelwert um $26 \%$ reduziert.

\section{Weichenstellung für eine erfolgreiche Transformation}

Für das Frühjahr plant der VOA eine erneute Umfrage unter seinen Mitgliedern. Es bleibt $\mathrm{zu}$ hoffen, dass die positive Entwicklung auch in Anbetracht des erneuten Lockdowns anhält insbesondere vor dem Hintergrund, dass über die wirtschaftlichen Auswirkungen der Pandemie hinaus wichtige Aufgaben anstehen. „Trotz der aktuell herausfordernden Pandemie-Situation dürfen wir andere drängende Themen nicht aus den Augen verlieren“, findet Dr.Alexa Becker, Geschäftsführerin des VOA. „Zum einen hat sich der Transformationsprozess der Industrie weltweit rasant beschleunigt. Zum anderen bringen die ambitionierten Klimaziele der Europäischen Kommission vor allem angesichts der Schwächephase der europäischen Wirtschaft, ausgelöst durch das Coronavirus, zusätzliche Belastungen - aber auch Chancen - für die Industrie mit sich.“
Gerade im Hinblick auf die Erfüllung der Vorgaben des European Green Deal und des Pariser Klimaschutzabkommens stehen Veränderungen an. Unternehmen sehen sich beispielsweise mit Themen wie Energieeinsparung, dem effizienten Einsatz von Ressourcen und der Reduzierung von Emissionen konfrontiert. Bislang fehle es aber an konkreten Maßnahmen, wie die europäische Industrie von Seiten der EU im Transformationsprozess unterstützt werden kann.

„Wirtschaftlichkeit und soziale Verträglichkeit sollten vor dem Hintergrund der Corona-Krise mehr denn je Hand in Hand gehen, denn nur eine starke und wettbewerbsfähige Industrie kann für einen wirtschaftlichen Aufschwung sorgen und die notwendigen Investitionen in Klimaund Umweltschutz erwirtschaften. Das ist eine echte Zukunftschance - auch für die Unternehmen in der Oberflächenveredelungsindustrie“, erklärt Dr. Becker. Für eine erfolgreiche Transformation gelte es, langfristige, industriepolitische Rahmenbedingungen zu schaffen. Zum Beispiel sei es sinnvoll, Innovationen und Forschung zu fördern, Energiepreise zu minimieren, Unternehmenssteuern zu senken und bürokratische Hürden zu beseitigen. //

\section{Kontakt}

VOA Verband für die Oberflächenveredelung von Aluminium e. $v$. München

Dr. Alexa A. Becker, Geschäftsführerin info@voa.de

www.voa.de 


\section{DÖRKEN}

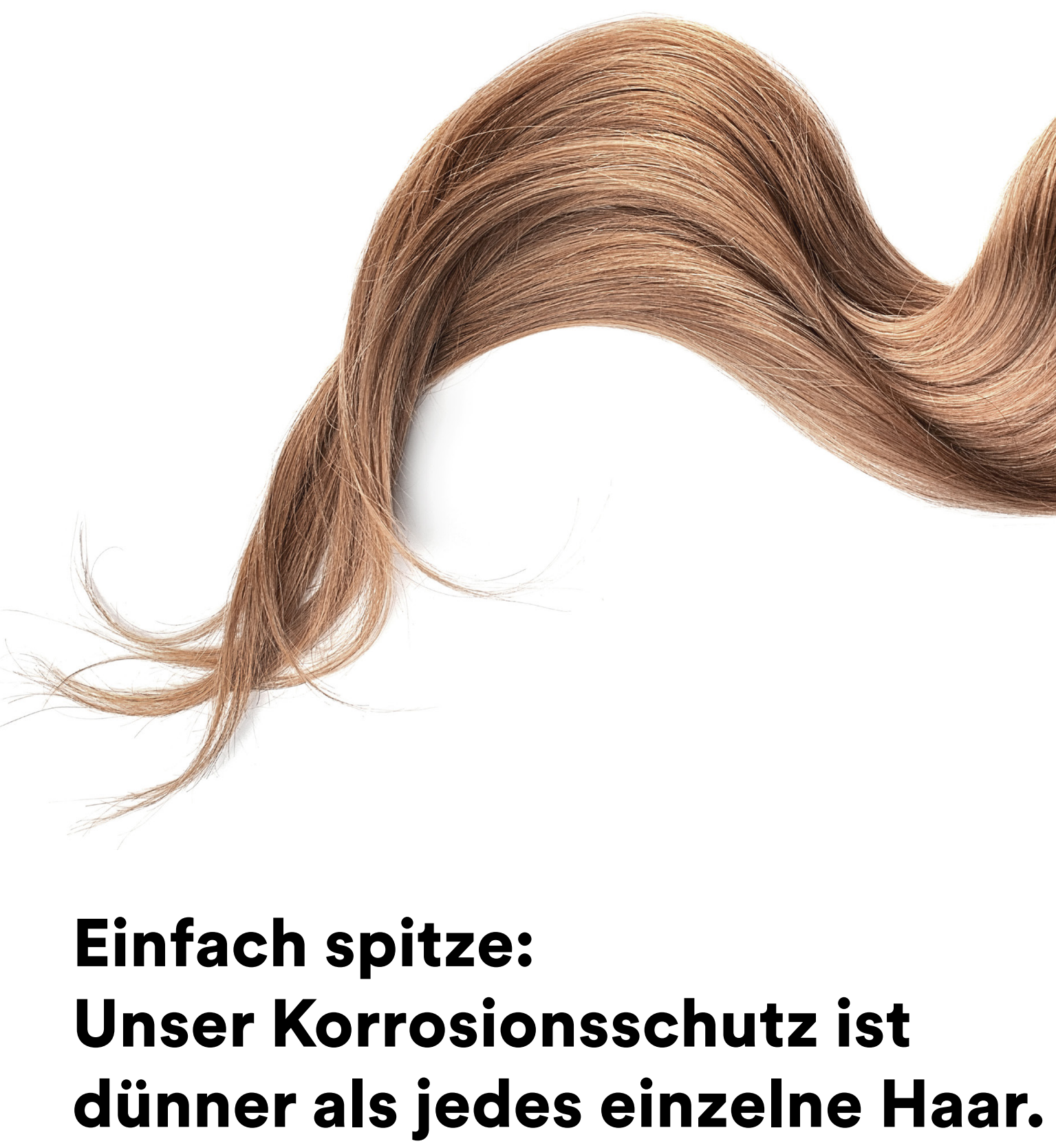

Und pflegeleichter sowieso! Trotz hauchdünner Schichtdicke erzielen unsere Beschichtungen ausgezeichnete Ergebnisse und bieten einen hohen Schutz. Und noch besser: Auch in Sachen Service scheren wir Sie nicht über einen Kamm, sondern gehen individuell auf Ihre Wünsche und Anforderungen ein.

www.doerken.de 